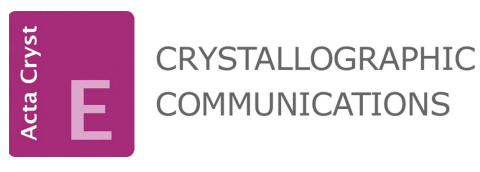

ISSN 2056-9890

Received 9 June 2016

Accepted 1 July 2016

Edited by M. Gdaniec, Adam Mickiewicz University, Poland

Keywords: crystal structure; copper; pyrazolate; trinuclear complex; terminal sulfate ligand.

CCDC reference: 1489622

Supporting information: this article has supporting information at journals.iucr.org/e

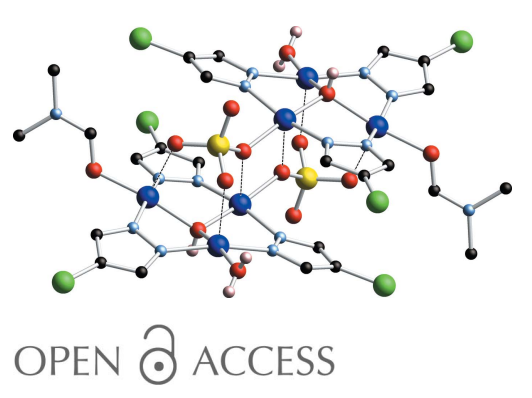

\section{Sulfate-bridged dimeric trinuclear copper(II)- pyrazolate complex with three different terminal ligands}

\author{
Gellert Mezei*
}

Department of Chemistry, Western Michigan University, Kalamazoo, Michigan, USA. *Correspondence e-mail: gellert.mezei@wmich.edu

The reaction of $\mathrm{CuSO}_{4} \cdot 5 \mathrm{H}_{2} \mathrm{O}, 4$-chloropyrazole (4-Cl-pzH) and triethylamine $\left(\mathrm{Et}_{3} \mathrm{~N}\right)$ in dimethylformamide $(\mathrm{DMF})$ produced crystals of diaquahexakis $(\mu-4-$ chloropyrazolato- $\left.\kappa^{2} N: N^{\prime}\right)$ bis $(N, N$-dimethylformamide $)$ di- $\mu_{3}$-hydroxido-bis $\left(\mu_{4}\right.$ sulfato- $\left.\kappa^{4} O: O^{\prime}: O^{\prime \prime}: O^{\prime \prime}\right)$ hexacopper(II) $N, N$-dimethylformamide tetrasolvate dihydrate, $\quad\left[\mathrm{Cu}_{3}(\mathrm{OH})\left(\mathrm{SO}_{4}\right)\left(\mathrm{C}_{3} \mathrm{H}_{2} \mathrm{ClN}_{2}\right)_{3}\left(\mathrm{C}_{3} \mathrm{H}_{7} \mathrm{NO}\right)\left(\mathrm{H}_{2} \mathrm{O}\right)\right]_{2} \cdot 4 \mathrm{C}_{3} \mathrm{H}_{7} \mathrm{NO} \cdot 2 \mathrm{H}_{2} \mathrm{O}$. The centrosymmetric dimeric molecule consists of two trinuclear copperpyrazolate units bridged by two sulfate ions. The title compound provides the first example of a trinuclear copper-pyrazolate complex with three different terminal ligands on the $\mathrm{Cu}$ atoms, and also the first example of such complex with a strongly binding basal sulfate ion. Within each trinuclear unit, the $\mathrm{Cu}^{\mathrm{II}}$ atoms are bridged by $\mu$-pyrazolate groups and a central $\mu_{3}-\mathrm{OH}$ group, and are coordinated by terminal sulfate, $\mathrm{H}_{2} \mathrm{O}$ and DMF ligands, respectively. Moreover, the sulfate $\mathrm{O}$ atoms coordinate at the apical position to the $\mathrm{Cu}$ atoms of the symmetry-related unit, providing square-pyramidal coordination geometry around each copper cation. The metal complex and solvent molecules are involved in $\mathrm{O}-\mathrm{H} \cdots \mathrm{O}$ hydrogen bonds, leading to a two-dimensional network parallel to $(10 \overline{1})$.

\section{Chemical context}

Trinuclear copper(II) complexes are primarily studied for their relevance to multicopper enzymes, such as oxidases (e.g., laccase, ascorbate oxidase, ceruloplasmin), oxygenases (e.g., tyrosinase, particulate methane monooxygenase, ammonia monooxygenase) and reductases (e.g., nitrite reductase, nitrous oxide reductase) (Solomon et al., 1996, 2014). Thus, such complexes are important targets from synthesis, redox chemistry and catalysis viewpoints (Di Nicola et al., 2009; Mimmi et al., 2004; Tsui et al., 2011; Lionetti et al., 2013; Grundner et al., 2015). Trinuclear copper(II) complexes also display interesting spectroscopic and magnetic properties (Boča et al., 2003; Rivera-Carrillo et al., 2008; Spielberg et al., 2015), and have been crucial in studying concepts such as spin frustration ( $\mathrm{Fu}$ et al., 2015). The pyrazolate anion is an excellent ligand for the construction of cyclic trinuclear and higher nuclearity metal complexes, leading to a variety of molecular architectures based on copper or other metals (Halcrow, 2009; Viciano-Chumillas et al., 2010).

A unique class of copper-pyrazolate complexes is defined by nanojars, based on a series of cyclic polymerization isomers, $\left[\text { cis- } \mathrm{Cu}^{\mathrm{II}}(\mu-\mathrm{OH})(\mu-\mathrm{pz})\right]_{n}(\mathrm{pz}=$ pyrazolate anion, $n=6-14$, except 11), which incarcerate anions with large hydration energies (e.g., sulfate, phosphate, carbonate) with unprecedented strength (Fernando et al., 2012; Mezei, 2015; Ahmed, 
Szymczyna et al., 2016) and permits the extraction of such anions from water into aliphatic solvents (Ahmed, Calco et al., 2016). Nanojars are obtained by self-assembly from a copper salt, pyrazole and a base (needed both for deprotonating pyrazole and as a hydroxide ion source) in the presence of an anion with large hydration energy, via a trinuclear intermediate, which is isolable and can be converted into nanojars by adding a base (Ahmed \& Mezei, 2016). Use of a strong base, such as sodium or tetrabutylammonium hydroxide, allows the preparation of nanojar solutions in different organic solvents. In contrast, a weak base, such as triethylamine, can only be employed as hydroxide source $\left(\mathrm{Et}_{3} \mathrm{~N}+\right.$ $\mathrm{H}_{2} \mathrm{O} \leftrightarrow \mathrm{Et}_{3} \mathrm{NH}^{+}+\mathrm{HO}^{-}$) if the nanojar product is precipitated out of the solution by dilution with excess water, in which the nanojar is not soluble (Fernando et al., 2012). Isolation of the title compound provides further evidence that in a neat organic solvent, such as $N, N$-dimethylformamide, the selfassembly process using triethylamine halts at the trinuclear stage, due to the acidity of the conjugate acid (triethylammonium cation, $\mathrm{p} K_{\mathrm{a}}=10.75$ in $\mathrm{H}_{2} \mathrm{O}$ ).

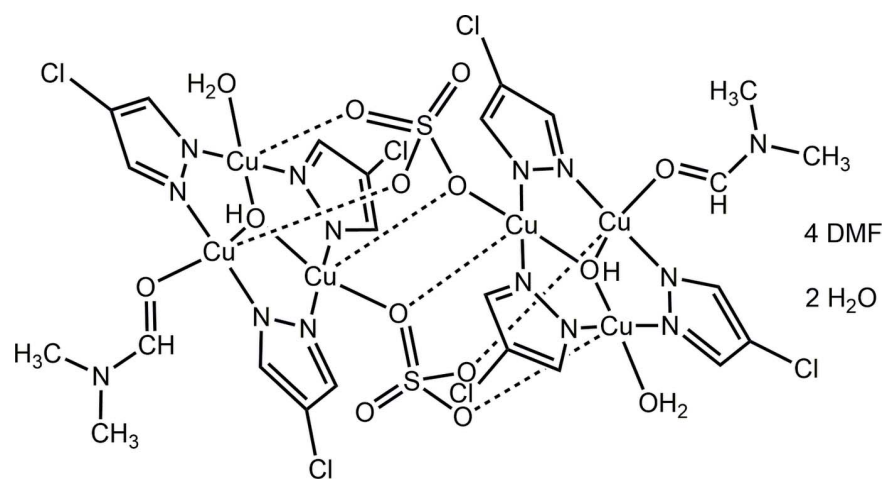

\section{Structural commentary}

The title metal complex molecule, located around an inversion center, consists of two symmetry-related trinuclear copper pyrazolate units (Fig. 1) connected together by sulfate ions

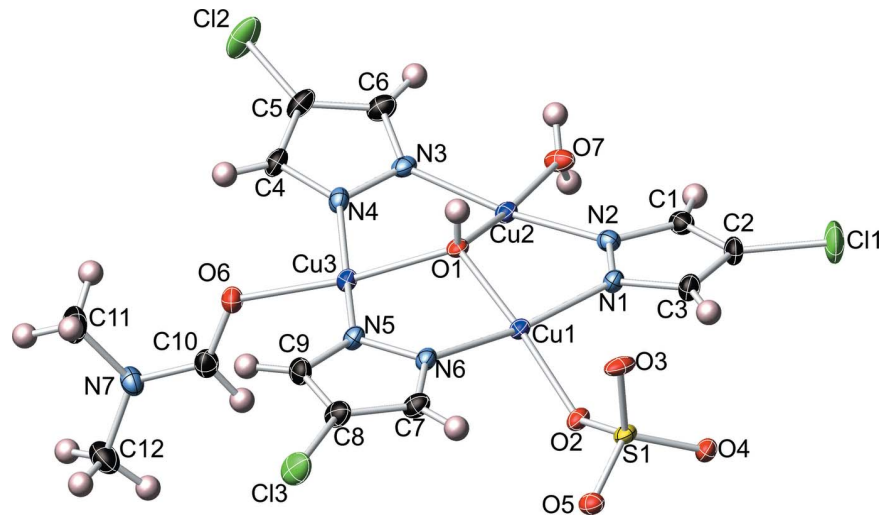

Figure 1

Displacement ellipsoid plot (50\% probability level) of the asymmetric unit of the title complex, showing the atom-labeling scheme (DMF and $\mathrm{H}_{2} \mathrm{O}$ solvent molecules omitted).
Table 1

Hydrogen-bond geometry $\left(\AA{ }^{\circ}\right)$.

\begin{tabular}{|c|c|c|c|c|}
\hline$D-\mathrm{H} \cdots A$ & $D-\mathrm{H}$ & $\mathrm{H} \cdots A$ & $D \cdots A$ & $D-\mathrm{H} \cdots A$ \\
\hline $\mathrm{C} 13-\mathrm{H} 13 \cdots \mathrm{O} 5^{\mathrm{i}}$ & 0.93 & 2.23 & $3.155(3)$ & 170 \\
\hline $\mathrm{C} 6-\mathrm{H} 6 \cdots \mathrm{O} 10^{\mathrm{ii}}$ & 0.93 & 2.38 & $3.234(4)$ & 153 \\
\hline $\mathrm{O} 10-\mathrm{H} 10 B \cdots \mathrm{O} 9^{\mathrm{iii}}$ & $0.81(2)$ & $1.96(2)$ & $2.751(3)$ & $165(4)$ \\
\hline $\mathrm{O} 10-\mathrm{H} 10 A \cdots \mathrm{O} 3^{\mathrm{iv}}$ & $0.81(2)$ & $1.91(2)$ & $2.700(3)$ & $165(4)$ \\
\hline $\mathrm{O} 7-\mathrm{H} 7 B \cdots{ }^{\mathrm{v}}$ & $0.83(2)$ & $1.83(2)$ & $2.658(3)$ & $175(3)$ \\
\hline $\mathrm{O} 7-\mathrm{H} 7 A \cdots \mathrm{O} 10^{\mathrm{ii}}$ & $0.80(2)$ & $1.83(2)$ & $2.625(3)$ & $172(3)$ \\
\hline $\mathrm{O} 1-\mathrm{H} 1 O \cdots \mathrm{O} 9^{\mathrm{vi}}$ & $0.78(2)$ & $1.95(2)$ & $2.711(3)$ & $166(3)$ \\
\hline $\mathrm{O} 1-\mathrm{H} 1 O \cdots \mathrm{O} 9^{\mathrm{vi}}$ & $0.78(2)$ & $1.95(2)$ & $2.711(3)$ & $166(3)$ \\
\hline $\mathrm{O} 7-\mathrm{H} 7 A \cdots \mathrm{O} 10^{\mathrm{ii}}$ & $0.80(2)$ & $1.83(2)$ & $2.625(3)$ & $172(3)$ \\
\hline $\mathrm{O} 7-\mathrm{H} 7 B \cdots \mathrm{O}^{\mathrm{v}}$ & $0.83(2)$ & $1.83(2)$ & $2.658(3)$ & $175(3)$ \\
\hline $\mathrm{O} 10-\mathrm{H} 10 A \cdots \mathrm{O} 3^{\text {iv }}$ & $0.81(2)$ & $1.91(2)$ & $2.700(3)$ & $165(4)$ \\
\hline $\mathrm{O} 10-\mathrm{H} 10 B \cdots \mathrm{O} 9^{\mathrm{iii}}$ & $0.81(2)$ & $1.96(2)$ & $2.751(3)$ & $165(4)$ \\
\hline
\end{tabular}

Symmetry codes: (i) $-x+1,-y+2,-z+1$; (ii) $-x+1,-y+1,-z+1$; (iii) $-x,-y+1,-z+1 ; \quad$ (iv) $\quad x-\frac{1}{2},-y+\frac{3}{2}, z-\frac{1}{2} ; \quad$ (v) $\quad x+1, y, z+1$; $-x+\frac{1}{2}, y+\frac{1}{2},-z+\frac{3}{2}$

(Fig. 2). One $\mathrm{O}$ atom of the sulfate moiety coordinates to one of the three independent $\mathrm{Cu}^{\mathrm{II}}$ atoms as basal donor $[\mathrm{Cu} 1-\mathrm{O} 2$ : 1.976 (2) $\AA$ ] , and to the corresponding symmetry-related $\mathrm{Cu}^{\text {II }}$ atom as apical donor [Cu1'-O2: 2.277 (2) $\AA$ ] . The other two $\mathrm{O}$ atoms of the sulfate moiety coordinate apically to the other two $\mathrm{Cu}$ atoms of the symmetry-related trinuclear unit, whereas the fourth $\mathrm{O}$ atom accepts a hydrogen bond from the solvent water molecule (Table 1). A square-pyramidal coordination geometry around each of the $\mathrm{Cu}^{\mathrm{II}}$ atoms is completed by the bridging $\mu$-pyrazolate and $\mu_{3}-\mathrm{OH}$ moieties, and terminal water or dimethylformamide molecules in basal positions. The $\mathrm{Cu}_{3}(\mu-4-\mathrm{Cl}-\mathrm{pz})_{3}$ core is relatively flat, with dihedral angles between the 4-chloropyrazolate mean planes and the $\mathrm{Cu}_{3}$ mean plane of $1.74(6), 7.20(6)$ and $14.10(4)^{\circ}$. The $\mu_{3}-\mathrm{OH}$ group is located $0.5615(15) \AA$ above the $\mathrm{Cu}_{3}$ mean plane. Bond lengths and angles within the $\mathrm{Cu}_{3}(\mu-4-\mathrm{Cl}-\mathrm{pz})_{3}$ framework are similar to the ones found in related complexes (Mezei et al., 2007; Rivera-Carrillo et al., 2008). The sulfatebridged dimeric structure presented here is reminiscent of dimeric trinuclear copper-pyrazolate complexes with bridging carboxylates (Mezei et al., 2004; Casarin et al., 2005).

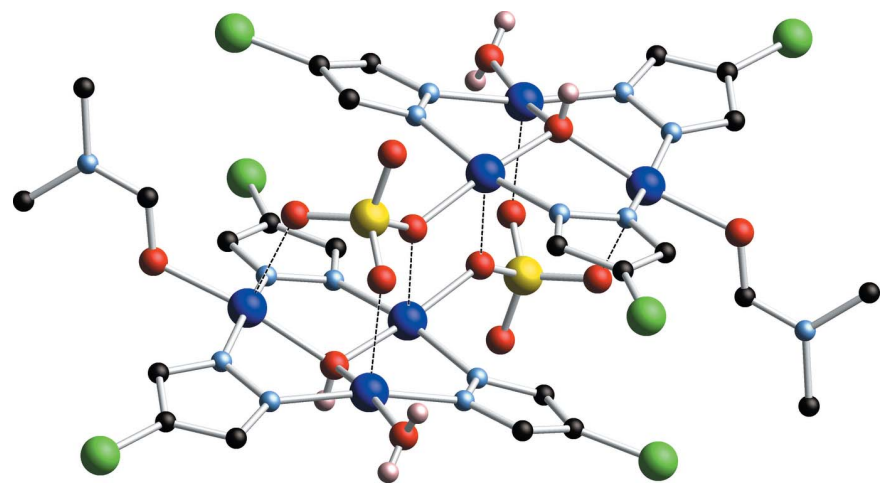

Figure 2

Dimeric structure formed by mutual apical coordination of three sulfate $\mathrm{O}$ atoms to the $\mathrm{Cu}$ atoms of the symmetry-related trinuclear copper(II)pyrazolate complex. 
Table 2

Experimental details.

\begin{tabular}{|c|c|}
\hline \multicolumn{2}{|l|}{ Crystal data } \\
\hline Chemical formula & $\begin{array}{l}{\left[\mathrm{Cu}_{6}(\mathrm{OH})_{2}\left(\mathrm{SO}_{4}\right)_{2}\left(\mathrm{C}_{3} \mathrm{H}_{2} \mathrm{ClN}_{2}\right)_{6-}\right.} \\
\left.\quad\left(\mathrm{C}_{3} \mathrm{H}_{7} \mathrm{NO}\right)_{2}\left(\mathrm{H}_{2} \mathrm{O}\right)_{2}\right] \cdot 4 \mathrm{C}_{3} \mathrm{H}_{7} \mathrm{NO} \cdot- \\
\quad 2 \mathrm{H}_{2} \mathrm{O}\end{array}$ \\
\hline$M_{\mathrm{r}}$ & 1727.11 \\
\hline Crystal system, space group & Monoclinic, $P 2_{1} / n$ \\
\hline Temperature $(\mathrm{K})$ & 100 \\
\hline$a, b, c(\AA)$ & $\begin{array}{l}12.7038(1), 16.5265(2), \\
16.6830(2)\end{array}$ \\
\hline$\beta\left(^{\circ}\right)$ & $109.774(1)$ \\
\hline$V\left(\AA^{3}\right)$ & $3296.05(6)$ \\
\hline$Z$ & 2 \\
\hline Radiation type & Мо $K \alpha$ \\
\hline$\mu\left(\mathrm{mm}^{-1}\right)$ & 2.29 \\
\hline Crystal size $(\mathrm{mm})$ & $0.24 \times 0.10 \times 0.05$ \\
\hline \multicolumn{2}{|l|}{ Data collection } \\
\hline Diffractometer & Bruker APEXII CCD \\
\hline Absorption correction & $\begin{array}{l}\text { Multi-scan (SADABS; Bruker, } \\
\text { 2014) }\end{array}$ \\
\hline$T_{\min }, T_{\max }$ & $0.610,0.894$ \\
\hline $\begin{array}{l}\text { No. of measured, independent and } \\
\text { observed }[I>2 \sigma(I)] \text { reflections }\end{array}$ & $39853,8504,6351$ \\
\hline$R_{\mathrm{int}}$ & 0.061 \\
\hline$(\sin \theta / \lambda)_{\max }\left(\AA^{-1}\right)$ & 0.676 \\
\hline \multicolumn{2}{|l|}{ Refinement } \\
\hline$R\left[F^{2}>2 \sigma\left(F^{2}\right)\right], w R\left(F^{2}\right), S$ & $0.035,0.075,1.01$ \\
\hline No. of reflections & 8504 \\
\hline No. of parameters & 418 \\
\hline No. of restraints & 5 \\
\hline H-atom treatment & $\begin{array}{l}\mathrm{H} \text { atoms treated by a mixture of } \\
\text { independent and constrained } \\
\text { refinement }\end{array}$ \\
\hline$\Delta \rho_{\max }, \Delta \rho_{\min }\left(\mathrm{e} \AA^{-3}\right)$ & $0.59,-0.52$ \\
\hline
\end{tabular}

Computer programs: APEX2 and SAINT (Bruker, 2014), SHELXS97 and SHELXTL (Sheldrick, 2008) and SHELXL2014 (Sheldrick, 2015).

\section{Supramolecular features}

The dimeric metal complex participates in an intricate hydrogen-bond network with the solvent DMF and $\mathrm{H}_{2} \mathrm{O}$ molecules. Numerical details of the hydrogen bonding are given in Table 1 . The $\mu_{3}-\mathrm{OH}$ group donates a hydrogen bond to a solvent DMF molecule [O1…O9: 2.711 (3) A], whereas the coordinating water molecule donates two hydrogen bonds, one to the solvent water molecule [O7...O10: 2.625 (3) A] and one to the other independent DMF solvent molecule [O7... O8: 2.658 (3) §]]. The solvent water molecule donates two hydrogen bonds, one to a sulfate $\mathrm{O}$ atom [O10...O3: 2.700 (3) $\AA$ ] and one to a DMF solvent molecule [O10 . . O9: 2.751 (3) $\AA$ ]. Within the dimeric unit, $\pi-\pi$ interactions are identified between pairs of pyrazolate moieties along the sulfate-bridged sides of the trinuclear units [centroid-centroid distance: 3.641 (1) $\AA$; dihedral angle: $7.5(1)^{\circ}$ ].

\section{Database survey}

A search of the Cambridge Structural Database (Groom et al., 2016) reveals only three trinuclear copper pyrazolate structures that contain sulfate (Zheng et al., 2008; Di Nicola et al., 2010). In all three cases, the sulfate ion coordinates weakly at the apical position of the copper cations $(\mathrm{Cu}-\mathrm{O}$ bonds lengths $>2.3 \AA$ ). Thus, the complex presented here is the first example of a trinuclear copper pyrazolate with the sulfate anion strongly binding at the basal position to a pentacoordinate $\mathrm{Cu}$-atom [Cu1-O2: 1.976 (2) $\AA$ ] .

\section{Synthesis and crystallization}

Copper sulfate pentahydrate $(1.000 \mathrm{~g})$, 4-chloropyrazole (411 mg) and $\mathrm{Et}_{3} \mathrm{~N}(1.2 \mathrm{~mL})$ were dissolved in DMF $(20 \mathrm{~mL})$ yielding a deep-blue solution. Dark-blue prismatic crystals of the title compound were obtained upon slow evaporation of the solvent.

\section{Refinement}

Crystal data, data collection and structure refinement details are summarized in Table 2 . $\mathrm{C}-\mathrm{H}$ hydrogen atoms were placed in idealized positions and refined using the riding-model approximation. The $\mathrm{OH}$ hydrogen atoms were located from difference Fourier maps; their displacement parameters were fixed to be $20 \%$ larger than those of the attached $\mathrm{O}$ atoms. $\mathrm{O}-\mathrm{H}$ distances were restrained to 0.82 (2) $\AA$.

\section{Acknowledgements}

This material is based upon work supported by the National Science Foundation under grant No. CHE-1404730.

\section{References}

Ahmed, B. M., Calco, B. \& Mezei, G. (2016). Dalton Trans. 45, 83278339.

Ahmed, B. M. \& Mezei, G. (2016). Inorg. Chem. Submitted.

Ahmed, B. M., Szymczyna, B. R., Jianrattanasawat, S., Surmann, S. A. \& Mezei, G. (2016). Chem. Eur. J. 22, 5499-5503.

Boča, R., Dlháň, L., Mezei, G., Ortiz-Pérez, T., Raptis, R. G. \& Telser, J. (2003). Inorg. Chem. 42, 5801-5803.

Bruker (2014). APEX2, SAINT and $S A D A B S$. Bruker AXS Inc., Madison, Wisconsin, USA.

Casarin, M., Corvaja, C., Di Nicola, C., Falcomer, D., Franco, L., Monari, M., Pandolfo, L., Pettinari, C. \& Piccinelli, F. (2005). Inorg. Chem. 44, 6265-6276.

Di Nicola, C., Garau, F., Gazzano, M., Monari, M., Pandolfo, L., Pettinari, C. \& Pettinari, R. (2010). Cryst. Growth Des. 10, 31203131.

Di Nicola, C., Garau, F., Karabach, Y. Y., Martins, L. M. D. R. S., Monari, M., Pandolfo, L., Pettinari, C. \& Pombeiro, A. J. L. (2009). Eur. J. Inorg. Chem. pp. 666-676.

Fernando, I. R., Surmann, S. A., Urech, A. A., Poulsen, A. M. \& Mezei, G. (2012). Chem. Commun. 48, 6860-6862.

Fu, M., Imai, T., Han, T.-H. \& Lee, Y. S. (2015). Science, 350, 655-658.

Groom, C. R., Bruno, I. J., Lightfoot, M. P. \& Ward, S. C. (2016). Acta Cryst. B72, 171-179.

Grundner, S., Markovits, M. A. C., Li, G., Tromp, M., Pidko, E. A., Hensen, E. J. M., Jentys, A., Sanchez-Sanchez, M. \& Lercher, J. A. (2015). Nat. Commun. 6, 7546.

Halcrow, M. A. (2009). Dalton Trans. pp. 2059-2073.

Lionetti, D., Day, M. W. \& Agapie, T. (2013). Chem. Sci. 4, 785-790.

Mezei, G. (2015). Chem. Commun. 51, 10341-10344.

Mezei, G., Rivera-Carrillo, M. \& Raptis, R. G. (2004). Inorg. Chim. Acta, 357, 3721-3732. 
Mezei, G., Rivera-Carrillo, M. \& Raptis, R. G. (2007). Dalton Trans. pp. 37-40.

Mimmi, M. C., Gullotti, M., Santagostini, L., Battaini, G., Monzani, E., Pagliarin, R., Zoppellaro, G. \& Casella, L. (2004). Dalton Trans. pp. 2192-2201.

Rivera-Carrillo, M., Chakraborty, I., Mezei, G., Webster, R. D. \& Raptis, R. G. (2008). Inorg. Chem. 47, 7644-7650.

Sheldrick, G. M. (2008). Acta Cryst. A64, 112-122.

Sheldrick, G. M. (2015). Acta Cryst. C71, 3-8.

Solomon, E. I., Heppner, D. E., Johnston, E. M., Ginsbach, J. W., Cirera, J., Qayyum, M., Kieber-Emmons, M. T., Kjaergaard, C. H., Hadt, R. G. \& Tian, L. (2014). Chem. Rev. 114, 3659-3853.
Solomon, E. I., Sundaram, U. M. \& Machonkin, T. E. (1996). Chem. Rev. 96, 2563-2606.

Spielberg, E. T., Gilb, A., Plaul, D., Geibig, D., Hornig, D., Schuch, D., Buchholz, A., Ardavan, A. \& Plass, W. (2015). Inorg. Chem. 54, 3432-3438.

Tsui, E. Y., Day, M. W. \& Agapie, T. (2011). Angew. Chem. Int. Ed. 50, 1668-1672.

Viciano-Chumillas, M., Tanase, S., de Jongh, L. J. \& Reedijk, J. (2010). Eur. J. Inorg. Chem. pp. 3403-3418.

Zheng, L.-L., Leng, J.-D., Zheng, S.-L., Zhaxi, Y.-C., Zhang, W.-X. \& Tong, M.-L. (2008). CrystEngComm, 10, 1467-1473. 


\section{supporting information}

Acta Cryst. (2016). E72, 1064-1067 [https://doi.org/10.1107/S2056989016010719]

\section{Sulfate-bridged dimeric trinuclear copper(II)-pyrazolate complex with three different terminal ligands}

\section{Gellert Mezei}

Computing details

Data collection: APEX2 (Bruker, 2014); cell refinement: SAINT (Bruker, 2014); data reduction: SAINT (Bruker, 2014); program(s) used to solve structure: SHELXS97 (Sheldrick, 2008); program(s) used to refine structure: SHELXL2014 (Sheldrick, 2015); molecular graphics: SHELXTL (Sheldrick, 2008); software used to prepare material for publication: SHELXTL (Sheldrick, 2008).

Diaquahexakis $\left(\mu\right.$-4-chloropyrazolato- $\left.\kappa^{2} N: N^{\prime}\right)$ bis $\left(N, N\right.$-dimethylformamide) di- $\mu_{3}$-hydroxido-bis $\left(\mu_{4}\right.$-sulfato$\left.\kappa^{4} O: O^{\prime}: O^{\prime \prime}: O^{\prime \prime}\right)$ hexacopper(II) $N, N$-dimethylformamide tetrasolvate dihydrate

Crystal data

$\left[\mathrm{Cu}_{6}(\mathrm{OH})_{2}\left(\mathrm{SO}_{4}\right)_{2}\left(\mathrm{C}_{3} \mathrm{H}_{2} \mathrm{ClN}_{2}\right)_{6}\left(\mathrm{C}_{3} \mathrm{H}_{7} \mathrm{NO}\right)_{2}\left(\mathrm{H}_{2} \mathrm{O}\right)_{2}\right] \cdot 4 \mathrm{C}_{3} \mathrm{H}_{7} \mathrm{NO} \cdot 2 \mathrm{H} 2 \mathrm{Q}=1.740 \mathrm{Mg} \mathrm{m}^{-3}$

$M_{r}=1727.11$

Monoclinic, $P 2_{1} / n$

$a=12.7038(1) \AA$

$b=16.5265(2) \AA$

$c=16.6830(2) \AA$

$\beta=109.774(1)^{\circ}$

$V=3296.05(6) \AA^{3}$

$Z=2$

$F(000)=1748$

Data collection

Bruker APEXII CCD

diffractometer

$\varphi$ and $\omega$ scans

Absorption correction: multi-scan

(SADABS; Bruker, 2014)

$T_{\min }=0.610, T_{\max }=0.894$

39853 measured reflections

Refinement

Refinement on $F^{2}$

Least-squares matrix: full

$R\left[F^{2}>2 \sigma\left(F^{2}\right)\right]=0.035$

$w R\left(F^{2}\right)=0.075$

$S=1.01$

8504 reflections

418 parameters

5 restraints
Mo $K \alpha$ radiation, $\lambda=0.71073 \AA$

Cell parameters from 6640 reflections

$\theta=2.6-26.9^{\circ}$

$\mu=2.29 \mathrm{~mm}^{-1}$

$T=100 \mathrm{~K}$

Prism, blue

$0.24 \times 0.10 \times 0.05 \mathrm{~mm}$

8504 independent reflections 6351 reflections with $I>2 \sigma(I)$

$R_{\text {int }}=0.061$

$\theta_{\text {max }}=28.7^{\circ}, \theta_{\min }=1.8^{\circ}$

$h=-17 \rightarrow 17$

$k=-20 \rightarrow 22$

$l=-22 \rightarrow 22$

Hydrogen site location: mixed

$\mathrm{H}$ atoms treated by a mixture of independent and constrained refinement

$w=1 /\left[\sigma^{2}\left(F_{\mathrm{o}}{ }^{2}\right)+(0.0294 P)^{2}+1.238 P\right]$

where $P=\left(F_{\mathrm{o}}^{2}+2 F_{\mathrm{c}}^{2}\right) / 3$

$(\Delta / \sigma)_{\max }=0.001$

$\Delta \rho_{\max }=0.59 \mathrm{e} \AA^{-3}$

$\Delta \rho_{\min }=-0.52$ e $\AA^{-3}$ 


\section{Special details}

Geometry. All esds (except the esd in the dihedral angle between two 1.s. planes) are estimated using the full covariance matrix. The cell esds are taken into account individually in the estimation of esds in distances, angles and torsion angles; correlations between esds in cell parameters are only used when they are defined by crystal symmetry. An approximate (isotropic) treatment of cell esds is used for estimating esds involving l.s. planes.

Fractional atomic coordinates and isotropic or equivalent isotropic displacement parameters $\left(\AA^{2}\right)$

\begin{tabular}{|c|c|c|c|c|}
\hline & $x$ & $y$ & $z$ & $U_{\text {iso }} * / U_{\text {eq }}$ \\
\hline $\mathrm{Cu} 1$ & $0.88533(2)$ & $0.98432(2)$ & $0.92266(2)$ & $0.01205(7)$ \\
\hline $\mathrm{Cu} 2$ & $0.84732(2)$ & $0.78607(2)$ & $0.89552(2)$ & $0.01339(8)$ \\
\hline $\mathrm{Cu} 3$ & $0.71256(3)$ & $0.89070(2)$ & $1.00006(2)$ & $0.01415(8)$ \\
\hline $\mathrm{S} 1$ & $1.02182(5)$ & $1.14500(4)$ & $0.90916(4)$ & $0.01287(13)$ \\
\hline $\mathrm{Cl1}$ & $1.14671(7)$ & $0.88248(5)$ & $0.71858(6)$ & $0.0362(2)$ \\
\hline $\mathrm{Cl} 2$ & $0.50045(6)$ & $0.57973(5)$ & $0.93424(6)$ & $0.0375(2)$ \\
\hline $\mathrm{Cl} 3$ & $0.69134(6)$ & $1.23152(4)$ & $1.07089(5)$ & $0.02872(17)$ \\
\hline $\mathrm{O} 1$ & $0.78020(14)$ & $0.89300(10)$ & $0.90866(11)$ & $0.0124(4)$ \\
\hline $\mathrm{H} 1 \mathrm{O}$ & $0.7316(19)$ & $0.8995(17)$ & $0.8661(13)$ & $0.015^{*}$ \\
\hline $\mathrm{O} 2$ & $1.00215(13)$ & $1.06890(10)$ & $0.95164(11)$ & $0.0137(4)$ \\
\hline $\mathrm{O} 3$ & $0.92608(14)$ & $1.15827(11)$ & $0.83199(12)$ & $0.0181(4)$ \\
\hline $\mathrm{O} 4$ & $1.12603(14)$ & $1.13455(11)$ & $0.89049(12)$ & $0.0162(4)$ \\
\hline $\mathrm{O} 5$ & $1.03357(14)$ & $1.21179(10)$ & $0.96983(11)$ & $0.0156(4)$ \\
\hline O6 & $0.62509(15)$ & $0.88937(11)$ & $1.07932(12)$ & $0.0193(4)$ \\
\hline $\mathrm{O} 7$ & $0.89793(15)$ & $0.68175(11)$ & $0.86138(13)$ & $0.0191(4)$ \\
\hline $\mathrm{H} 7 \mathrm{~A}$ & $0.855(2)$ & $0.6528(16)$ & $0.8274(16)$ & $0.023 *$ \\
\hline H7B & $0.9493(19)$ & $0.6535(16)$ & $0.8930(17)$ & $0.023 *$ \\
\hline O8 & $0.07007(16)$ & $0.59491(12)$ & $-0.04174(13)$ & $0.0265(5)$ \\
\hline O9 & $-0.13195(15)$ & $0.43846(12)$ & $0.74393(13)$ & $0.0264(5)$ \\
\hline $\mathrm{O} 10$ & $0.24177(18)$ & $0.42323(14)$ & $0.23718(17)$ & $0.0412(7)$ \\
\hline $\mathrm{H} 10 \mathrm{~A}$ & $0.293(2)$ & $0.401(2)$ & $0.2728(19)$ & $0.049 *$ \\
\hline $\mathrm{H} 10 \mathrm{~B}$ & $0.219(3)$ & $0.4655(15)$ & $0.251(2)$ & $0.049 *$ \\
\hline N1 & $0.95307(17)$ & $0.92644(13)$ & $0.85102(14)$ & $0.0147(5)$ \\
\hline N2 & $0.94773(17)$ & $0.84403(13)$ & $0.84797(14)$ & $0.0149(5)$ \\
\hline N3 & $0.72096(17)$ & $0.73695(13)$ & $0.92013(14)$ & $0.0155(5)$ \\
\hline N4 & $0.67343(17)$ & $0.77874(13)$ & $0.96960(14)$ & $0.0154(5)$ \\
\hline N5 & $0.72004(17)$ & $1.00879(13)$ & $1.00420(14)$ & $0.0156(5)$ \\
\hline N6 & $0.79401(16)$ & $1.04688(13)$ & $0.97334(14)$ & $0.0142(5)$ \\
\hline N7 & $0.61860(18)$ & $0.90095(14)$ & $1.21195(15)$ & $0.0201(5)$ \\
\hline N8 & 0.21667 (19) & $0.62870(14)$ & $0.07710(15)$ & $0.0218(5)$ \\
\hline N9 & $0.03464(18)$ & $0.37758(13)$ & $0.81585(15)$ & $0.0195(5)$ \\
\hline $\mathrm{C} 1$ & $1.0125(2)$ & $0.81766(16)$ & $0.80436(17)$ & $0.0175(6)$ \\
\hline $\mathrm{H} 1$ & 1.0244 & 0.7638 & 0.7937 & $0.021 *$ \\
\hline $\mathrm{C} 2$ & $1.0585(2)$ & $0.88365(17)$ & $0.77797(18)$ & $0.0196(6)$ \\
\hline C3 & $1.0202(2)$ & $0.95107(17)$ & $0.80841(17)$ & $0.0183(6)$ \\
\hline $\mathrm{H} 3$ & 1.0378 & 1.0045 & 0.8008 & $0.022 *$ \\
\hline $\mathrm{C} 4$ & $0.5943(2)$ & $0.73193(16)$ & $0.98217(18)$ & $0.0185(6)$ \\
\hline $\mathrm{H} 4 \mathrm{~A}$ & 0.5498 & 0.7459 & 1.0143 & $0.022 *$ \\
\hline $\mathrm{C} 5$ & $0.5894(2)$ & $0.65981(16)$ & 0.93968 (19) & $0.0207(6)$ \\
\hline
\end{tabular}




$\begin{array}{lllll}\text { C6 } & 0.6705(2) & 0.66438(16) & 0.90177(19) & 0.0201(6) \\ \text { H6 } & 0.6874 & 0.6241 & 0.8691 & 0.024^{*} \\ \text { C7 } & 0.7943(2) & 1.12579(16) & 0.99150(18) & 0.0175(6) \\ \text { H7 } & 0.8377 & 1.1651 & 0.9777 & 0.021^{*} \\ \text { C8 } & 0.7200(2) & 1.13943(16) & 1.03396(18) & 0.0192(6) \\ \text { C9 } & 0.6751(2) & 1.06498(16) & 1.04087(18) & 0.0187(6) \\ \text { H9 } & 0.6221 & 1.0552 & 1.0668 & 0.022^{*} \\ \text { C10 } & 0.6727(2) & 0.89642(16) & 1.15752(19) & 0.0199(6) \\ \text { H10 } & 0.7504 & 0.8986 & 1.1785 & 0.024^{*} \\ \text { C11 } & 0.4967(2) & 0.8972(2) & 1.1816(2) & 0.0321(8) \\ \text { H11A } & 0.4714 & 0.8602 & 1.1347 & 0.048^{*} \\ \text { H11B } & 0.4667 & 0.9500 & 1.1634 & 0.048^{*} \\ \text { H11C } & 0.4719 & 0.8790 & 1.2269 & 0.048^{*} \\ \text { C12 } & 0.6772(3) & 0.9130(2) & 1.30266(19) & 0.0300(7) \\ \text { H12A } & 0.6561 & 0.9643 & 1.3197 & 0.045^{*} \\ \text { H12B } & 0.7565 & 0.9124 & 1.3138 & 0.045^{*} \\ \text { H12C } & 0.6578 & 0.8705 & 1.3342 & 0.045^{*} \\ \text { C13 } & 0.1149(2) & 0.63767(17) & 0.02161(19) & 0.0216(6) \\ \text { H13 } & 0.0728 & 0.6803 & 0.0310 & 0.026^{*} \\ \text { C14 } & 0.2914(3) & 0.5656(2) & 0.0675(2) & 0.0379(8) \\ \text { H14A } & 0.2527 & 0.5324 & 0.0193 & 0.057^{*} \\ \text { H14B } & 0.3153 & 0.5328 & 0.1180 & 0.057^{*} \\ \text { H14C } & 0.3554 & 0.5899 & 0.0589 & 0.057^{*} \\ \text { C15 } & 0.2608(3) & 0.6836(2) & 0.1482(2) & 0.0341(8) \\ \text { H15A } & 0.2041 & 0.7218 & 0.1485 & 0.051^{*} \\ \text { H15B } & 0.3241 & 0.7119 & 0.1428 & 0.051^{*} \\ \text { H15C } & 0.2836 & 0.6534 & 0.2005 & 0.051^{*} \\ \text { C16 } & -0.0465(2) & 0.43014(17) & 0.80778(19) & 0.0225(6) \\ \text { H16 } & -0.0392 & 0.4640 & 0.8539 & 0.027^{*} \\ \text { C17 } & 0.1313(2) & 0.37298(19) & 0.89417(19) & 0.0273(7) \\ \text { H17A } & 0.1206 & 0.4090 & 0.9359 & 0.041^{*} \\ \text { H17B } & 0.1975 & 0.3884 & 0.8824 & 0.041^{*} \\ \text { H17C } & 0.1394 & 0.3186 & 0.9156 & 0.041^{*} \\ \text { C18 } & 0.0309(2) & 0.32090(18) & 0.7482(2) & 0.0278(7) \\ \text { H18A } & -0.0347 & 0.3310 & 0.6997 & 0.042^{*} \\ \text { H18B } & 0.0286 & 0.2666 & 0.7679 & 0.042^{*} \\ \text { H18C } & 0.0963 & 0.3277 & 0.7324 & 0.042^{*} \\ & & & & \end{array}$

Atomic displacement parameters $\left(\AA^{2}\right)$

\begin{tabular}{lllllll}
\hline & $U^{11}$ & $U^{22}$ & $U^{33}$ & $U^{12}$ & $U^{13}$ & $U^{23}$ \\
\hline $\mathrm{Cu} 1$ & $0.00914(14)$ & $0.01448(16)$ & $0.01225(16)$ & $-0.00096(12)$ & $0.00324(12)$ & $-0.00052(13)$ \\
$\mathrm{Cu} 2$ & $0.01008(14)$ & $0.01517(16)$ & $0.01420(17)$ & $-0.00047(12)$ & $0.00315(12)$ & $-0.00099(13)$ \\
$\mathrm{Cu} 3$ & $0.01137(15)$ & $0.01748(17)$ & $0.01439(17)$ & $-0.00170(12)$ & $0.00540(13)$ & $-0.00102(13)$ \\
$\mathrm{S} 1$ & $0.0095(3)$ & $0.0152(3)$ & $0.0120(3)$ & $-0.0015(2)$ & $0.0013(2)$ & $0.0007(2)$ \\
$\mathrm{C} 11$ & $0.0364(4)$ & $0.0423(5)$ & $0.0444(5)$ & $0.0025(4)$ & $0.0326(4)$ & $-0.0004(4)$ \\
$\mathrm{Cl} 2$ & $0.0273(4)$ & $0.0200(4)$ & $0.0715(7)$ & $-0.0091(3)$ & $0.0247(4)$ & $-0.0017(4)$ \\
$\mathrm{Cl} 3$ & $0.0227(3)$ & $0.0219(4)$ & $0.0440(5)$ & $0.0035(3)$ & $0.0146(3)$ & $-0.0092(3)$
\end{tabular}




\begin{tabular}{|c|c|c|c|c|c|c|}
\hline $\mathrm{O} 1$ & $0.0079(8)$ & $0.0164(9)$ & $0.0111(10)$ & $-0.0004(7)$ & $0.0010(7)$ & $-0.0014(8)$ \\
\hline $\mathrm{O} 2$ & $0.0103(8)$ & $0.0159(9)$ & $0.0139(10)$ & $-0.0013(7)$ & $0.0025(7)$ & $0.0031(7)$ \\
\hline $\mathrm{O} 3$ & $0.0127(9)$ & $0.0206(10)$ & $0.0144(10)$ & $-0.0021(7)$ & $-0.0039(8)$ & $0.0042(8)$ \\
\hline $\mathrm{O} 4$ & $0.0118(8)$ & $0.0215(10)$ & $0.0156(10)$ & $-0.0023(7)$ & $0.0052(8)$ & $-0.0007(8)$ \\
\hline $\mathrm{O} 5$ & $0.0130(8)$ & $0.0163(9)$ & $0.0156(10)$ & $-0.0003(7)$ & $0.0024(7)$ & $-0.0019(8)$ \\
\hline O6 & $0.0172(9)$ & $0.0256(11)$ & $0.0172(11)$ & $-0.0035(8)$ & $0.0088(8)$ & $-0.0015(8)$ \\
\hline $\mathrm{O} 7$ & $0.0145(9)$ & $0.0183(10)$ & 0.0205 (11) & $0.0018(7)$ & $0.0006(8)$ & $-0.0050(8)$ \\
\hline $\mathrm{O} 8$ & $0.0224(10)$ & $0.0286(11)$ & $0.0237(12)$ & $0.0038(9)$ & $0.0014(9)$ & $-0.0041(9)$ \\
\hline O9 & $0.0201(10)$ & $0.0254(11)$ & $0.0236(12)$ & $0.0033(8)$ & $-0.0059(9)$ & $-0.0051(9)$ \\
\hline $\mathrm{O} 10$ & $0.0244(12)$ & $0.0337(14)$ & $0.0449(16)$ & $0.0136(10)$ & $-0.0152(11)$ & $-0.0228(12)$ \\
\hline N1 & $0.0117(10)$ & $0.0180(11)$ & $0.0149(12)$ & $-0.0020(8)$ & $0.0053(9)$ & $-0.0007(9)$ \\
\hline N2 & $0.0133(10)$ & $0.0160(11)$ & $0.0149(12)$ & $0.0002(8)$ & $0.0043(9)$ & $-0.0030(9)$ \\
\hline N3 & $0.0117(10)$ & $0.0182(12)$ & $0.0145(12)$ & $-0.0015(8)$ & $0.0015(9)$ & $0.0001(9)$ \\
\hline N4 & $0.0115(10)$ & $0.0198(12)$ & $0.0159(12)$ & $0.0005(9)$ & $0.0060(9)$ & $0.0016(9)$ \\
\hline N5 & $0.0115(10)$ & $0.0185(12)$ & $0.0172(12)$ & $-0.0012(8)$ & $0.0054(9)$ & $-0.0005(9)$ \\
\hline N6 & $0.0102(10)$ & $0.0181(11)$ & $0.0142(12)$ & $-0.0011(8)$ & $0.0040(9)$ & $0.0005(9)$ \\
\hline N7 & $0.0206(12)$ & $0.0241(13)$ & $0.0179(13)$ & $0.0045(10)$ & $0.0094(10)$ & $0.0009(10)$ \\
\hline N8 & $0.0191(12)$ & $0.0230(13)$ & $0.0195(13)$ & $0.0034(10)$ & $0.0015(10)$ & $0.0023(10)$ \\
\hline N9 & 0.0155 (11) & $0.0210(12)$ & 0.0177 (13) & $-0.0006(9)$ & $-0.0002(10)$ & $0.0024(10)$ \\
\hline $\mathrm{C} 1$ & $0.0143(12)$ & 0.0209 (14) & $0.0164(14)$ & $0.0012(10)$ & $0.0039(11)$ & $-0.0029(11)$ \\
\hline $\mathrm{C} 2$ & 0.0148 (13) & $0.0284(15)$ & $0.0189(15)$ & $0.0016(11)$ & $0.0101(12)$ & $-0.0021(12)$ \\
\hline $\mathrm{C} 3$ & 0.0155 (13) & $0.0226(14)$ & $0.0188(15)$ & -0.0024 & $0.0084(11)$ & 0.0005 (11) \\
\hline $\mathrm{C} 4$ & $0.0122(12)$ & $0.0206(14)$ & $0.0238(16)$ & $-0.0019(10)$ & $0.0075(11)$ & 0.0015 (11) \\
\hline $\mathrm{C} 5$ & $0.0135(12)$ & $0.0168(14)$ & $0.0310(17)$ & $-0.0026(10)$ & 0.0065 (12) & $0.0027(12)$ \\
\hline C6 & $0.0163(13)$ & $0.0164(14)$ & $0.0254(16)$ & $0.0002(10)$ & $0.0040(12)$ & $0.0000(12)$ \\
\hline $\mathrm{C} 7$ & $0.0131(12)$ & 0.0157 (13) & $0.0219(15)$ & $0.0007(10)$ & $0.0036(11)$ & $-0.0001(11)$ \\
\hline $\mathrm{C} 8$ & $0.0138(12)$ & $0.0187(14)$ & $0.0248(16)$ & $0.0031(10)$ & $0.0061(12)$ & $-0.0033(12)$ \\
\hline C9 & $0.0150(13)$ & $0.0237(15)$ & $0.0191(15)$ & $0.0034(11)$ & 0.0077 (11) & $-0.0014(12)$ \\
\hline $\mathrm{C} 10$ & $0.0197(14)$ & $0.0207(14)$ & $0.0226(16)$ & $-0.0017(11)$ & $0.0114(12)$ & $-0.0013(12)$ \\
\hline C11 & $0.0202(15)$ & $0.052(2)$ & $0.0276(18)$ & $0.0047(14)$ & $0.0125(14)$ & $0.0045(15)$ \\
\hline C12 & $0.0308(16)$ & 0.0405 (19) & 0.0193 (16) & $0.0059(14)$ & 0.0093 (13) & -0.0009 (14) \\
\hline C13 & $0.0174(13)$ & $0.0245(15)$ & $0.0217(16)$ & $0.0035(11)$ & $0.0051(12)$ & $0.0018(12)$ \\
\hline $\mathrm{C} 14$ & $0.0251(16)$ & $0.039(2)$ & $0.043(2)$ & $0.0143(14)$ & $0.0026(15)$ & $0.0005(16)$ \\
\hline $\mathrm{C} 15$ & $0.0299(17)$ & $0.0330(18)$ & 0.0291 (19) & $-0.0012(14)$ & $-0.0036(14)$ & $-0.0036(15)$ \\
\hline $\mathrm{C} 16$ & $0.0213(14)$ & $0.0230(15)$ & $0.0206(16)$ & $-0.0041(11)$ & 0.0035 (12) & $-0.0012(12)$ \\
\hline $\mathrm{C} 17$ & 0.0187 (14) & $0.0349(18)$ & $0.0221(17)$ & $-0.0027(12)$ & $-0.0013(12)$ & $0.0097(13)$ \\
\hline $\mathrm{C} 18$ & $0.0240(15)$ & $0.0244(16)$ & 0.0324 (19) & $0.0040(12)$ & 0.0063 (14) & $0.0025(13)$ \\
\hline
\end{tabular}

Geometric parameters $\left(\AA,{ }^{\circ}\right)$

\begin{tabular}{lllr}
\hline $\mathrm{Cu} 1-\mathrm{N} 1$ & $1.944(2)$ & $\mathrm{N} 7-\mathrm{C} 10$ & $1.313(3)$ \\
$\mathrm{Cu} 1-\mathrm{N} 6$ & $1.948(2)$ & $\mathrm{N} 7-\mathrm{C} 12$ & $1.457(4)$ \\
$\mathrm{Cu} 1-\mathrm{O} 2$ & $1.9760(17)$ & $\mathrm{N} 7-\mathrm{C} 11$ & $1.458(3)$ \\
$\mathrm{Cu} 1-\mathrm{O} 1$ & $1.9761(17)$ & $\mathrm{N} 8-\mathrm{C} 13$ & $1.319(3)$ \\
$\mathrm{Cu} 1-\mathrm{O} 2$ & $\mathrm{~N} 8-\mathrm{C} 15$ & $1.447(4)$ \\
$\mathrm{Cu} 2-\mathrm{N} 3$ & $2.2773(17)$ & $\mathrm{N} 8-\mathrm{C} 14$ & $1.455(4)$ \\
$\mathrm{Cu} 2-\mathrm{N} 2$ & $1.962(2)$ & $\mathrm{N} 9-\mathrm{C} 16$ & $1.320(3)$ \\
$\mathrm{Cu} 2-\mathrm{O} 7$ & $1.964(2)$ & $\mathrm{N} 9-\mathrm{C} 18$ & $1.455(4)$
\end{tabular}




\begin{tabular}{|c|c|c|c|}
\hline $\mathrm{Cu} 2-\mathrm{O} 1$ & $2.0061(17)$ & N9- $\mathrm{C} 17$ & $1.461(3)$ \\
\hline $\mathrm{Cu} 2-\mathrm{O}^{\mathrm{i}}$ & $2.2444(18)$ & $\mathrm{C} 1-\mathrm{C} 2$ & $1.378(4)$ \\
\hline $\mathrm{Cu} 3-\mathrm{N} 4$ & $1.939(2)$ & $\mathrm{C} 1-\mathrm{H} 1$ & 0.9300 \\
\hline $\mathrm{Cu} 3-\mathrm{N} 5$ & $1.954(2)$ & $\mathrm{C} 2-\mathrm{C} 3$ & $1.380(4)$ \\
\hline $\mathrm{Cu} 3-\mathrm{O} 1$ & $1.9879(18)$ & $\mathrm{C} 3-\mathrm{H} 3$ & 0.9300 \\
\hline $\mathrm{Cu} 3-\mathrm{O} 6$ & $1.9945(18)$ & $\mathrm{C} 4-\mathrm{C} 5$ & 1.377 (4) \\
\hline $\mathrm{Cu} 3-\mathrm{O} 4^{\mathrm{i}}$ & $2.2759(18)$ & $\mathrm{C} 4-\mathrm{H} 4 \mathrm{~A}$ & 0.9300 \\
\hline $\mathrm{S} 1-\mathrm{O} 3$ & $1.4579(18)$ & $\mathrm{C} 5-\mathrm{C} 6$ & $1.382(4)$ \\
\hline $\mathrm{S} 1-\mathrm{O} 4$ & $1.4691(18)$ & C6-H6 & 0.9300 \\
\hline $\mathrm{S} 1-\mathrm{O} 5$ & $1.4708(18)$ & $\mathrm{C} 7-\mathrm{C} 8$ & $1.377(4)$ \\
\hline $\mathrm{S} 1-\mathrm{O} 2$ & $1.5055(18)$ & $\mathrm{C} 7-\mathrm{H} 7$ & 0.9300 \\
\hline $\mathrm{C} 11-\mathrm{C} 2$ & $1.729(3)$ & $\mathrm{C} 8-\mathrm{C} 9$ & $1.377(4)$ \\
\hline $\mathrm{Cl} 2-\mathrm{C} 5$ & $1.723(3)$ & C9- $\mathrm{H} 9$ & 0.9300 \\
\hline $\mathrm{Cl} 3-\mathrm{C} 8$ & $1.726(3)$ & $\mathrm{C} 10-\mathrm{H} 10$ & 0.9300 \\
\hline $\mathrm{O} 1-\mathrm{H} 1 \mathrm{O}$ & $0.775(17)$ & $\mathrm{C} 11-\mathrm{H} 11 \mathrm{~A}$ & 0.9600 \\
\hline $\mathrm{O} 2-\mathrm{Cu} 1^{\mathrm{i}}$ & $2.2774(17)$ & $\mathrm{C} 11-\mathrm{H} 11 \mathrm{~B}$ & 0.9600 \\
\hline $\mathrm{O} 4-\mathrm{Cu}^{\mathrm{i}}$ & $2.2759(18)$ & $\mathrm{C} 11-\mathrm{H} 11 \mathrm{C}$ & 0.9600 \\
\hline $\mathrm{O} 5-\mathrm{Cu} 2^{\mathrm{i}}$ & $2.2444(18)$ & $\mathrm{C} 12-\mathrm{H} 12 \mathrm{~A}$ & 0.9600 \\
\hline $\mathrm{O} 6-\mathrm{C} 10$ & $1.244(3)$ & $\mathrm{C} 12-\mathrm{H} 12 \mathrm{~B}$ & 0.9600 \\
\hline $\mathrm{O} 7-\mathrm{H} 7 \mathrm{~A}$ & 0.801 (17) & $\mathrm{C} 12-\mathrm{H} 12 \mathrm{C}$ & 0.9600 \\
\hline $\mathrm{O} 7-\mathrm{H} 7 \mathrm{~B}$ & 0.831 (17) & $\mathrm{C} 13-\mathrm{H} 13$ & 0.9300 \\
\hline $\mathrm{O} 8-\mathrm{C} 13$ & $1.238(3)$ & $\mathrm{C} 14-\mathrm{H} 14 \mathrm{~A}$ & 0.9600 \\
\hline $\mathrm{O} 9-\mathrm{C} 16$ & $1.245(3)$ & C14-H14B & 0.9600 \\
\hline $\mathrm{O} 10-\mathrm{H} 10 \mathrm{~A}$ & 0.808 (18) & $\mathrm{C} 14-\mathrm{H} 14 \mathrm{C}$ & 0.9600 \\
\hline $\mathrm{O} 10-\mathrm{H} 10 \mathrm{~B}$ & 0.814 (18) & C15-H15A & 0.9600 \\
\hline $\mathrm{N} 1-\mathrm{C} 3$ & $1.345(3)$ & C15-H15B & 0.9600 \\
\hline $\mathrm{N} 1-\mathrm{N} 2$ & $1.364(3)$ & $\mathrm{C} 15-\mathrm{H} 15 \mathrm{C}$ & 0.9600 \\
\hline $\mathrm{N} 2-\mathrm{C} 1$ & $1.342(3)$ & C16-H16 & 0.9300 \\
\hline N3-C6 & $1.345(3)$ & C17-H17A & 0.9600 \\
\hline N3-N4 & $1.364(3)$ & C17-H17B & 0.9600 \\
\hline $\mathrm{N} 4-\mathrm{C} 4$ & $1.340(3)$ & $\mathrm{C} 17-\mathrm{H} 17 \mathrm{C}$ & 0.9600 \\
\hline N5-C9 & $1.341(3)$ & C18-H18A & 0.9600 \\
\hline N5-N6 & $1.368(3)$ & C18-H18B & 0.9600 \\
\hline $\mathrm{N} 6-\mathrm{C} 7$ & $1.339(3)$ & $\mathrm{C} 18-\mathrm{H} 18 \mathrm{C}$ & 0.9600 \\
\hline $\mathrm{N} 1-\mathrm{Cu} 1-\mathrm{N} 6$ & $168.69(9)$ & $\mathrm{C} 16-\mathrm{N} 9-\mathrm{C} 18$ & $121.7(2)$ \\
\hline $\mathrm{N} 1-\mathrm{Cu} 1-\mathrm{O} 2$ & $92.66(8)$ & $\mathrm{C} 16-\mathrm{N} 9-\mathrm{C} 17$ & $121.1(3)$ \\
\hline $\mathrm{N} 6-\mathrm{Cu} 1-\mathrm{O} 2$ & $91.53(8)$ & $\mathrm{C} 18-\mathrm{N} 9-\mathrm{C} 17$ & $117.2(2)$ \\
\hline $\mathrm{N} 1-\mathrm{Cu} 1-\mathrm{O} 1$ & $88.49(8)$ & $\mathrm{N} 2-\mathrm{C} 1-\mathrm{C} 2$ & $108.7(2)$ \\
\hline $\mathrm{N} 6-\mathrm{Cu} 1-\mathrm{O} 1$ & $88.78(8)$ & $\mathrm{N} 2-\mathrm{C} 1-\mathrm{H} 1$ & 125.7 \\
\hline $\mathrm{O} 2-\mathrm{Cu} 1-\mathrm{O} 1$ & $172.22(7)$ & $\mathrm{C} 2-\mathrm{C} 1-\mathrm{H} 1$ & 125.7 \\
\hline $\mathrm{N} 1-\mathrm{Cu} 1-\mathrm{O} 2^{\mathrm{i}}$ & $96.06(8)$ & $\mathrm{C} 1-\mathrm{C} 2-\mathrm{C} 3$ & $106.3(2)$ \\
\hline $\mathrm{N} 6-\mathrm{Cu} 1-\mathrm{O} 2^{\mathrm{i}}$ & $94.92(8)$ & $\mathrm{C} 1-\mathrm{C} 2-\mathrm{C} 11$ & $127.0(2)$ \\
\hline $\mathrm{O} 2-\mathrm{Cu} 1-\mathrm{O} 2^{\mathrm{i}}$ & $82.09(7)$ & $\mathrm{C} 3-\mathrm{C} 2-\mathrm{C} 11$ & $126.7(2)$ \\
\hline $\mathrm{O} 1-\mathrm{Cu} 1-\mathrm{O} 2^{\mathrm{i}}$ & $90.14(7)$ & $\mathrm{N} 1-\mathrm{C} 3-\mathrm{C} 2$ & $108.4(2)$ \\
\hline $\mathrm{N} 3-\mathrm{Cu} 2-\mathrm{N} 2$ & $167.22(9)$ & $\mathrm{N} 1-\mathrm{C} 3-\mathrm{H} 3$ & 125.8 \\
\hline $\mathrm{N} 3-\mathrm{Cu} 2-\mathrm{O} 7$ & $93.89(8)$ & $\mathrm{C} 2-\mathrm{C} 3-\mathrm{H} 3$ & 125.8 \\
\hline $\mathrm{N} 2-\mathrm{Cu} 2-\mathrm{O} 7$ & $89.43(8)$ & $\mathrm{N} 4-\mathrm{C} 4-\mathrm{C} 5$ & $108.9(2)$ \\
\hline
\end{tabular}




\begin{tabular}{|c|c|c|c|}
\hline $\mathrm{N} 3-\mathrm{Cu} 2-\mathrm{O} 1$ & $86.18(8)$ & $\mathrm{N} 4-\mathrm{C} 4-\mathrm{H} 4 \mathrm{~A}$ & 125.5 \\
\hline $\mathrm{N} 2-\mathrm{Cu} 2-\mathrm{O} 1$ & $88.44(8)$ & $\mathrm{C} 5-\mathrm{C} 4-\mathrm{H} 4 \mathrm{~A}$ & 125.5 \\
\hline $\mathrm{O} 7-\mathrm{Cu} 2-\mathrm{O} 1$ & $170.11(8)$ & $\mathrm{C} 4-\mathrm{C} 5-\mathrm{C} 6$ & $106.1(2)$ \\
\hline $\mathrm{N} 3-\mathrm{Cu} 2-\mathrm{O} 5^{\mathrm{i}}$ & $96.85(8)$ & $\mathrm{C} 4-\mathrm{C} 5-\mathrm{Cl} 2$ & $127.3(2)$ \\
\hline $\mathrm{N} 2-\mathrm{Cu} 2-\mathrm{O}^{\mathrm{i}}$ & $94.96(8)$ & $\mathrm{C} 6-\mathrm{C} 5-\mathrm{Cl} 2$ & $126.6(2)$ \\
\hline $\mathrm{O} 7-\mathrm{Cu} 2-\mathrm{O} 5^{\mathrm{i}}$ & $97.26(7)$ & $\mathrm{N} 3-\mathrm{C} 6-\mathrm{C} 5$ & $108.4(2)$ \\
\hline $\mathrm{O} 1-\mathrm{Cu} 2-\mathrm{O} 5^{\mathrm{i}}$ & $92.54(7)$ & $\mathrm{N} 3-\mathrm{C} 6-\mathrm{H} 6$ & 125.8 \\
\hline $\mathrm{N} 4-\mathrm{Cu} 3-\mathrm{N} 5$ & $165.39(9)$ & $\mathrm{C} 5-\mathrm{C} 6-\mathrm{H} 6$ & 125.8 \\
\hline $\mathrm{N} 4-\mathrm{Cu} 3-\mathrm{O} 1$ & $87.49(8)$ & $\mathrm{N} 6-\mathrm{C} 7-\mathrm{C} 8$ & $109.0(2)$ \\
\hline $\mathrm{N} 5-\mathrm{Cu} 3-\mathrm{O} 1$ & $88.83(8)$ & $\mathrm{N} 6-\mathrm{C} 7-\mathrm{H} 7$ & 125.5 \\
\hline $\mathrm{N} 4-\mathrm{Cu} 3-\mathrm{O} 6$ & $90.70(8)$ & $\mathrm{C} 8-\mathrm{C} 7-\mathrm{H} 7$ & 125.5 \\
\hline $\mathrm{N} 5-\mathrm{Cu} 3-\mathrm{O} 6$ & $91.08(8)$ & $\mathrm{C} 7-\mathrm{C} 8-\mathrm{C} 9$ & $105.8(2)$ \\
\hline $\mathrm{O} 1-\mathrm{Cu} 3-\mathrm{O} 6$ & $172.37(7)$ & $\mathrm{C} 7-\mathrm{C} 8-\mathrm{Cl} 3$ & $126.3(2)$ \\
\hline $\mathrm{N} 4-\mathrm{Cu} 3-\mathrm{O} 4^{\mathrm{i}}$ & $96.70(8)$ & $\mathrm{C} 9-\mathrm{C} 8-\mathrm{Cl} 3$ & $127.9(2)$ \\
\hline $\mathrm{N} 5-\mathrm{Cu} 3-\mathrm{O} 4^{\mathrm{i}}$ & $97.76(8)$ & $\mathrm{N} 5-\mathrm{C} 9-\mathrm{C} 8$ & $109.2(2)$ \\
\hline $\mathrm{O} 1-\mathrm{Cu} 3-\mathrm{O} 4^{\mathrm{i}}$ & $96.49(7)$ & $\mathrm{N} 5-\mathrm{C} 9-\mathrm{H} 9$ & 125.4 \\
\hline $\mathrm{O} 6-\mathrm{Cu} 3-\mathrm{O} 4^{\mathrm{i}}$ & $91.08(7)$ & $\mathrm{C} 8-\mathrm{C} 9-\mathrm{H} 9$ & 125.4 \\
\hline $\mathrm{O} 3-\mathrm{S} 1-\mathrm{O} 4$ & $111.90(11)$ & $\mathrm{O} 6-\mathrm{C} 10-\mathrm{N} 7$ & $123.2(3)$ \\
\hline $\mathrm{O} 3-\mathrm{S} 1-\mathrm{O} 5$ & $110.78(11)$ & $\mathrm{O} 6-\mathrm{C} 10-\mathrm{H} 10$ & 118.4 \\
\hline $\mathrm{O} 4-\mathrm{S} 1-\mathrm{O} 5$ & $110.20(10)$ & $\mathrm{N} 7-\mathrm{C} 10-\mathrm{H} 10$ & 118.4 \\
\hline $\mathrm{O} 3-\mathrm{S} 1-\mathrm{O} 2$ & $108.64(10)$ & N7-C11-H11A & 109.5 \\
\hline $\mathrm{O} 4-\mathrm{S} 1-\mathrm{O} 2$ & $107.86(10)$ & N7-C11-H11B & 109.5 \\
\hline $\mathrm{O} 5-\mathrm{S} 1-\mathrm{O} 2$ & $107.30(10)$ & $\mathrm{H} 11 \mathrm{~A}-\mathrm{C} 11-\mathrm{H} 11 \mathrm{~B}$ & 109.5 \\
\hline $\mathrm{Cu} 1-\mathrm{O} 1-\mathrm{Cu} 3$ & $111.97(8)$ & N7-C11-H11C & 109.5 \\
\hline $\mathrm{Cu} 1-\mathrm{O} 1-\mathrm{Cu} 2$ & $112.98(8)$ & $\mathrm{H} 11 \mathrm{~A}-\mathrm{C} 11-\mathrm{H} 11 \mathrm{C}$ & 109.5 \\
\hline $\mathrm{Cu} 3-\mathrm{O} 1-\mathrm{Cu} 2$ & $112.15(8)$ & $\mathrm{H} 11 \mathrm{~B}-\mathrm{C} 11-\mathrm{H} 11 \mathrm{C}$ & 109.5 \\
\hline $\mathrm{Cu} 1-\mathrm{O} 1-\mathrm{H} 1 \mathrm{O}$ & $107(2)$ & $\mathrm{N} 7-\mathrm{C} 12-\mathrm{H} 12 \mathrm{~A}$ & 109.5 \\
\hline $\mathrm{Cu} 3-\mathrm{O} 1-\mathrm{H} 1 \mathrm{O}$ & $107(2)$ & N7-C12-H12B & 109.5 \\
\hline $\mathrm{Cu} 2-\mathrm{O} 1-\mathrm{H} 1 \mathrm{O}$ & $105(2)$ & $\mathrm{H} 12 \mathrm{~A}-\mathrm{C} 12-\mathrm{H} 12 \mathrm{~B}$ & 109.5 \\
\hline $\mathrm{S} 1-\mathrm{O} 2-\mathrm{Cu} 1$ & $134.93(11)$ & $\mathrm{N} 7-\mathrm{C} 12-\mathrm{H} 12 \mathrm{C}$ & 109.5 \\
\hline $\mathrm{S} 1-\mathrm{O} 2-\mathrm{Cu} 1^{\mathrm{i}}$ & $127.14(10)$ & $\mathrm{H} 12 \mathrm{~A}-\mathrm{C} 12-\mathrm{H} 12 \mathrm{C}$ & 109.5 \\
\hline $\mathrm{Cu} 1-\mathrm{O} 2-\mathrm{Cu} 1^{\mathrm{i}}$ & $97.91(7)$ & $\mathrm{H} 12 \mathrm{~B}-\mathrm{C} 12-\mathrm{H} 12 \mathrm{C}$ & 109.5 \\
\hline $\mathrm{S} 1-\mathrm{O} 4-\mathrm{Cu} 3^{\mathrm{i}}$ & $118.91(11)$ & $\mathrm{O} 8-\mathrm{C} 13-\mathrm{N} 8$ & $126.4(3)$ \\
\hline $\mathrm{S} 1-\mathrm{O} 5-\mathrm{Cu} 2^{\mathrm{i}}$ & $125.29(10)$ & $\mathrm{O} 8-\mathrm{C} 13-\mathrm{H} 13$ & 116.8 \\
\hline $\mathrm{C} 10-\mathrm{O} 6-\mathrm{Cu} 3$ & $120.80(17)$ & $\mathrm{N} 8-\mathrm{C} 13-\mathrm{H} 13$ & 116.8 \\
\hline $\mathrm{Cu} 2-\mathrm{O} 7-\mathrm{H} 7 \mathrm{~A}$ & $121(2)$ & $\mathrm{N} 8-\mathrm{C} 14-\mathrm{H} 14 \mathrm{~A}$ & 109.5 \\
\hline $\mathrm{Cu} 2-\mathrm{O} 7-\mathrm{H} 7 \mathrm{~B}$ & $125(2)$ & $\mathrm{N} 8-\mathrm{C} 14-\mathrm{H} 14 \mathrm{~B}$ & 109.5 \\
\hline $\mathrm{H} 7 \mathrm{~A}-\mathrm{O} 7-\mathrm{H} 7 \mathrm{~B}$ & $108(3)$ & $\mathrm{H} 14 \mathrm{~A}-\mathrm{C} 14-\mathrm{H} 14 \mathrm{~B}$ & 109.5 \\
\hline $\mathrm{H} 10 \mathrm{~A}-\mathrm{O} 10-\mathrm{H} 10 \mathrm{~B}$ & $118(4)$ & $\mathrm{N} 8-\mathrm{C} 14-\mathrm{H} 14 \mathrm{C}$ & 109.5 \\
\hline $\mathrm{C} 3-\mathrm{N} 1-\mathrm{N} 2$ & $108.4(2)$ & $\mathrm{H} 14 \mathrm{~A}-\mathrm{C} 14-\mathrm{H} 14 \mathrm{C}$ & 109.5 \\
\hline $\mathrm{C} 3-\mathrm{N} 1-\mathrm{Cu} 1$ & $131.89(19)$ & $\mathrm{H} 14 \mathrm{~B}-\mathrm{C} 14-\mathrm{H} 14 \mathrm{C}$ & 109.5 \\
\hline $\mathrm{N} 2-\mathrm{N} 1-\mathrm{Cu} 1$ & $119.18(16)$ & $\mathrm{N} 8-\mathrm{C} 15-\mathrm{H} 15 \mathrm{~A}$ & 109.5 \\
\hline $\mathrm{C} 1-\mathrm{N} 2-\mathrm{N} 1$ & $108.2(2)$ & $\mathrm{N} 8-\mathrm{C} 15-\mathrm{H} 15 \mathrm{~B}$ & 109.5 \\
\hline $\mathrm{C} 1-\mathrm{N} 2-\mathrm{Cu} 2$ & $131.42(18)$ & $\mathrm{H} 15 \mathrm{~A}-\mathrm{C} 15-\mathrm{H} 15 \mathrm{~B}$ & 109.5 \\
\hline $\mathrm{N} 1-\mathrm{N} 2-\mathrm{Cu} 2$ & $120.19(15)$ & $\mathrm{N} 8-\mathrm{C} 15-\mathrm{H} 15 \mathrm{C}$ & 109.5 \\
\hline $\mathrm{C} 6-\mathrm{N} 3-\mathrm{N} 4$ & $108.4(2)$ & $\mathrm{H} 15 \mathrm{~A}-\mathrm{C} 15-\mathrm{H} 15 \mathrm{C}$ & 109.5 \\
\hline $\mathrm{C} 6-\mathrm{N} 3-\mathrm{Cu} 2$ & $132.94(19)$ & $\mathrm{H} 15 \mathrm{~B}-\mathrm{C} 15-\mathrm{H} 15 \mathrm{C}$ & 109.5 \\
\hline $\mathrm{N} 4-\mathrm{N} 3-\mathrm{Cu} 2$ & $118.57(16)$ & $\mathrm{O} 9-\mathrm{C} 16-\mathrm{N} 9$ & $125.8(3)$ \\
\hline
\end{tabular}




\begin{tabular}{llll} 
C4-N4-N3 & $108.2(2)$ & O9-C16-H16 & 117.1 \\
C4-N4-Cu3 & $130.49(18)$ & N9-C16-H16 & 117.1 \\
N3-N4-Cu3 & $121.01(15)$ & N9-C17-H17A & 109.5 \\
C9-N5-N6 & $107.8(2)$ & N9-C17-H17B & 109.5 \\
C9-N5-Cu3 & $133.20(18)$ & H17A-C17-H17B & 109.5 \\
N6-N5-Cu3 & $118.53(16)$ & N9-C17-H17C & 109.5 \\
C7-N6-N5 & $108.2(2)$ & H17A-C17-H17C & 109.5 \\
C7-N6-Cu1 & $131.22(18)$ & H17B-C17-H17C & 109.5 \\
N5-N6-Cu1 & $120.33(16)$ & N9-C18-H18A & 109.5 \\
C10-N7-C12 & $121.5(2)$ & N9-C18-H18B & 109.5 \\
C10-N7-C11 & $120.0(2)$ & H18A-C18-H18B & 109.5 \\
C12-N7-C11 & $118.4(2)$ & N9-C18-H18C & 109.5 \\
C13-N8-C15 & $121.4(2)$ & H18A-C18-H18C & 109.5 \\
C13-N8-C14 & $121.6(3)$ & H18B-C18-H18C & 109.5 \\
C15-N8-C14 & $116.9(2)$ & & \\
\hline
\end{tabular}

Symmetry code: (i) $-x+2,-y+2,-z+2$.

Hydrogen-bond geometry $\left(\AA,{ }^{\circ}\right)$

\begin{tabular}{|c|c|c|c|c|}
\hline$D-\mathrm{H} \cdots A$ & $D-\mathrm{H}$ & $\mathrm{H} \cdots A$ & $D \cdots A$ & $D-\mathrm{H} \cdots A$ \\
\hline $\mathrm{C} 18-\mathrm{H} 18 B \cdots \mathrm{O} 3^{\mathrm{ii}}$ & 0.96 & 2.64 & $3.494(4)$ & 148 \\
\hline $\mathrm{C} 17-\mathrm{H} 17 C \cdots \mathrm{O} 5^{\mathrm{ii}}$ & 0.96 & 2.56 & $3.360(3)$ & 141 \\
\hline $\mathrm{C} 15-\mathrm{H} 15 C \cdots \mathrm{N} 2^{\mathrm{iii}}$ & 0.96 & 2.63 & $3.406(4)$ & 138 \\
\hline $\mathrm{C} 13-\mathrm{H} 13 \cdots \mathrm{O}^{\mathrm{iv}}$ & 0.93 & 2.23 & $3.155(3)$ & 170 \\
\hline $\mathrm{C} 10-\mathrm{H} 10 \cdots \mathrm{O}^{\mathrm{i}}$ & 0.93 & 2.30 & $2.971(3)$ & 128 \\
\hline $\mathrm{C} 7-\mathrm{H} 7 \cdots \mathrm{O} 5$ & 0.93 & 2.65 & $3.483(3)$ & 149 \\
\hline $\mathrm{C} 7-\mathrm{H} 7 \cdots \mathrm{S} 1$ & 0.93 & 2.95 & $3.610(3)$ & 129 \\
\hline $\mathrm{C} 6-\mathrm{H} 6 \cdots \mathrm{O} 10^{v}$ & 0.93 & 2.38 & $3.234(4)$ & 153 \\
\hline $\mathrm{C} 4-\mathrm{H} 4 A \cdots \mathrm{Cl} 3^{\mathrm{vi}}$ & 0.93 & 2.93 & $3.484(3)$ & 119 \\
\hline $\mathrm{C} 3-\mathrm{H} 3 \cdots \mathrm{O} 4$ & 0.93 & 2.64 & $3.411(3)$ & 140 \\
\hline $\mathrm{C} 3-\mathrm{H} 3 \cdots \mathrm{S} 1$ & 0.93 & 2.99 & $3.616(3)$ & 126 \\
\hline $\mathrm{O} 10-\mathrm{H} 10 B \cdots \mathrm{O} 9^{\text {vii }}$ & $0.81(2)$ & $1.96(2)$ & $2.751(3)$ & $165(4)$ \\
\hline $\mathrm{O} 10-\mathrm{H} 10 A \cdots \mathrm{O} 3^{\mathrm{iii}}$ & $0.81(2)$ & $1.91(2)$ & $2.700(3)$ & $165(4)$ \\
\hline $\mathrm{O} 7-\mathrm{H} 7 B \cdots \mathrm{O} 8^{\text {viii }}$ & $0.83(2)$ & $1.83(2)$ & $2.658(3)$ & $175(3)$ \\
\hline $\mathrm{O} 7-\mathrm{H} 7 A \cdots \mathrm{O} 10^{\mathrm{v}}$ & $0.80(2)$ & $1.83(2)$ & $2.625(3)$ & $172(3)$ \\
\hline $\mathrm{O} 1-\mathrm{H} 1 O \cdots \mathrm{O} 9^{\text {ix }}$ & $0.78(2)$ & $1.95(2)$ & $2.711(3)$ & $166(3)$ \\
\hline $\mathrm{O} 1-\mathrm{H} 1 O \cdots \mathrm{O}^{\mathrm{ix}}$ & $0.78(2)$ & $1.95(2)$ & $2.711(3)$ & $166(3)$ \\
\hline $\mathrm{O} 7-\mathrm{H} 7 A \cdots \mathrm{O} 10^{\mathrm{v}}$ & $0.80(2)$ & $1.83(2)$ & $2.625(3)$ & $172(3)$ \\
\hline $\mathrm{O} 7-\mathrm{H} 7 B \cdots \mathrm{O} 8^{\mathrm{viii}}$ & $0.83(2)$ & $1.83(2)$ & $2.658(3)$ & $175(3)$ \\
\hline $\mathrm{O} 10-\mathrm{H} 10 A \cdots \mathrm{O} 3^{\mathrm{iii}}$ & $0.81(2)$ & $1.91(2)$ & $2.700(3)$ & $165(4)$ \\
\hline $\mathrm{O} 10-\mathrm{H} 10 B \cdots \mathrm{O} 9^{\text {vii }}$ & $0.81(2)$ & $1.96(2)$ & $2.751(3)$ & $165(4)$ \\
\hline $\mathrm{C} 3-\mathrm{H} 3 \cdots \mathrm{S} 1$ & 0.93 & 2.99 & $3.616(3)$ & 126 \\
\hline $\mathrm{C} 3-\mathrm{H} 3 \cdots \mathrm{O} 4$ & 0.93 & 2.64 & $3.411(3)$ & 140 \\
\hline $\mathrm{C} 4-\mathrm{H} 4 A \cdots \mathrm{Cl} 3^{\mathrm{vi}}$ & 0.93 & 2.93 & $3.484(3)$ & 119 \\
\hline $\mathrm{C} 6-\mathrm{H} 6 \cdots \mathrm{O} 10^{\mathrm{v}}$ & 0.93 & 2.38 & $3.234(4)$ & 153 \\
\hline $\mathrm{C} 7-\mathrm{H} 7 \cdots \mathrm{S} 1$ & 0.93 & 2.95 & $3.610(3)$ & 129 \\
\hline $\mathrm{C} 7-\mathrm{H} 7 \cdots \mathrm{O} 5$ & 0.93 & 2.65 & $3.483(3)$ & 149 \\
\hline
\end{tabular}


supporting information

$\begin{array}{lllll}\mathrm{C} 10-\mathrm{H} 10 \cdots \mathrm{O} 4^{\mathrm{i}} & 0.93 & 2.30 & 2.971(3) & 128 \\ \mathrm{C} 13-\mathrm{H} 13 \cdots \mathrm{O} 5^{\text {iv }} & 0.93 & 2.23 & 3.155(3) & 170 \\ \mathrm{C} 15-\mathrm{H} 15 C \cdots \mathrm{N} 2^{\mathrm{iii}} & 0.96 & 2.63 & 3.406(4) & 138 \\ \mathrm{C} 17-\mathrm{H} 17 C \cdots \mathrm{O} 5^{\mathrm{ii}} & 0.96 & 2.56 & 3.360(3) & 141 \\ \mathrm{C} 18-\mathrm{H} 18 B^{\cdots} \cdots 3^{3 i} & 0.96 & 2.64 & 3.494(4) & 148\end{array}$

Symmetry codes: (i) $-x+2,-y+2,-z+2$; (ii) $x-1, y-1, z$; (iii) $x-1 / 2,-y+3 / 2, z-1 / 2$; (iv) $-x+1,-y+2,-z+1$; (v) $-x+1,-y+1,-z+1$; (vi) $-x+1,-y+2,-z+2$; (vii) $-x,-y+1,-z+1$; (viii) $x+1, y, z+1$; (ix) $-x+1 / 2, y+1 / 2,-z+3 / 2$. 